\title{
Evolución de las capacidades físicas en jugadores jóvenes de baloncesto de medio nivel
}

\section{Evolution of physical characteristics in mid level young basketball players}

\author{
Evolução de habilidades físicas na juventude \\ jogadores de basquetebol de nível médio
}

\author{
Julio Calleja-González ${ }^{1}$, Jesús Cámara Tobalina ${ }^{1}$, Raúl Martínez-Santos ${ }^{1}$, Gaizka Mejuto ${ }^{1}$ y Nicolás Terrados ${ }^{2}$ \\ 1 Departamento de Educación Física y Deportiva, Facultad de Ciencias de la Actividad Física y el Deporte, Universidad del País Vasco (UPVIEHU), Vitoria \\ y 2 Unidad Regional de Medicina del Deporte y Departamento de Biologia Funcional. Universidad de Oviedo, Asturias.
}

\begin{abstract}
Resumen: objetivo: El objetivo de estudio fue analizar la evolución de las capacidades físicas de jugadores de medio nivel a lo largo de 2 años (categoría: cadete y júnior). Metodo. 15 jugadores fueron evaluados durante 2 ańos: en el ultimo ańo de la etapa cadete (U16) y el primer año de la etapa junior (U18). Los tests fueron realizados en 4 momentos (M) de cada temporada [1. Septiembre (pre-temporada), 2. Diciembre, 3. Abril y 4. Julio, para un total de 8 pruebas]. La batería consistió en un test de $20 \mathrm{~m}$ sprint (MST), un test de agilidad T (AGIT), un lanzamiento de balón medicinal de $5 \mathrm{~kg}$ (5KGT), un salto (CMJ), un test Sit and Reach (SAR) y la Course Navette $(\mathrm{CN})$. Resultados. Los principales resultados fueron:,los jugadores mejoraron en MST: [1० M: $3.16 \pm 0.27 \mathrm{~m} / \mathrm{s}$ Vs. $\left.4^{\circ} \mathrm{M}: 2.87 \pm 0.17 \mathrm{~m} / \mathrm{s} ;(\mathrm{p}<0.05)\right]$; en CMJ, observamos diferencias estadísticas entre el $1^{\circ}$ y $8^{\circ} \mathrm{M}\left[1^{\circ} \mathrm{M}: 32.57 \pm 2.8\right.$ $\mathrm{cm} \mathrm{Vs.} 8^{\circ} \mathrm{M}: 42.14 \pm 3.8 \mathrm{~cm}$; $\left.(\mathrm{p}<0.05)\right]$; los resultados en SAR mostraron que los valores decrecieron ( $1^{\circ} \mathrm{M}: 13.14 \pm 12.27 \mathrm{~cm}$ al $8^{\circ} \mathrm{M}: 6.42 \pm 7.36$ $\mathrm{cm}$ ); finalmente, observamos diferencias significativas entre $1^{\circ} \mathrm{M}$ y el $8^{\circ} \mathrm{M}$ en CNT [ $1^{\circ} \mathrm{M}: 10.36 \pm 1.7$ paliers Vs. $8^{\circ} \mathrm{M}: 12.5 \pm 1.4$ paliers; $\left.(\mathrm{p}<0.05)\right]$. Conclusiones. Se constataron mejoras significativas en las capacidades neuromusculares, la potencia aeróbica y la flexibilidad.

Palabras clave: baloncesto, capacidades físicas, jóvenes

Abstract: Aim: The aim of the study was to analyse the evolution of physical abilities of mid level basketball players during a 2 year period (age groups: cadet and junior). Method. 15 male basketball players were observed during 2 years: in the last year of cadet age group (U16) and in the first year of junior age group (U18). The test trials were performed 4 times (M) along the season. [1. September (pre-season), 2. December, 3. April and 4. July, for 8 different tests in total]. The test set was as follows: 20 $\mathrm{m}$ sprint test (MST), agility test (AGIT), medicinal ball throwing $5 \mathrm{~kg}$ (5KGT), vertical jump (CMJ), flexibility Sit and Reach test (SAR) and the Course Navette $(\mathrm{CN})$. Results. The players improved their MST: [1 ${ }^{\circ} \mathrm{M}$ :
\end{abstract}

$3.16 \pm 0.27 \mathrm{~m} / \mathrm{s}$ Vs. $\left.4^{\circ} \mathrm{M}: 2.87 \pm 0.17 \mathrm{~m} / \mathrm{s} ;(\mathrm{p}<0.05)\right]$; in CMJ, statistical differences were observed between $1^{\circ}$ and $8^{\circ} \mathrm{M}\left[1^{\circ} \mathrm{M}: 32.57 \pm 2.8 \mathrm{~cm} \mathrm{Vs}\right.$. $\left.8^{\circ} \mathrm{M}: 42.14 \pm 3.8 \mathrm{~cm} ;(\mathrm{p}<0.05)\right]$; the results of SAR test showed a decrease $\left(1^{\circ} \mathrm{M}: 13.14 \pm 12.27 \mathrm{~cm}\right.$ to $\left.8^{\circ} \mathrm{M}: 6.42 \pm 7.36 \mathrm{~cm}\right)$; other significant differences were also oberved when $1^{\circ} \mathrm{M}$ and $8^{\circ} \mathrm{M}$ were compared for CNT [1 ${ }^{\circ}$ M: $10.36 \pm 1.7$ stages $V_{s} .8^{\circ} \mathrm{M}: 12.5 \pm 1.4$ stages; $\left.(\mathrm{p}<0.05)\right]$. Conclusions. Significant improvements were unvealed for neuromuscular ability, aerobic power and flexibility.

Key words: basketball, physical characteristics, young players.

Resumo: Objetivo: O objetivo deste estudo foi analisar a evolução das capacidades físicas de jogadores de nível médio mais de dois anos (categoria: cadete e júnior). Método. 15 jogadores foram testados por 2 anos no último ano do cadete fase (U16) e do primeiro ano do estágio junior (U18). Os testes foram realizados em 4 momentos $(\mathrm{M})$ de cada estação [1. Setembro (pré-temporada), 02 de dezembro, 03 de abril, e 4. Julho, para um total de 8 testes]. A bateria consistiu de um teste de $20 \mathrm{~m}$ de sprint (MST), um teste de agilidade T (STIR) Um lance bola de medicina de $5 \mathrm{~kg}$ (5KGT), um salto (CMJ), um teste de sentar e alcançar (SAR) e o Curso Navette (CN). Resultados. Os principais resultados foram: os jogadores melhoraram no MST: [1 M: 3,16 $\pm 0,27 \mathrm{~m} / \mathrm{s}$ vs. $4 \mathrm{M}: 2,87 \pm 0,17 \mathrm{~m} / \mathrm{s}$; $(\mathrm{P}<0,05)]$; no CMJ, observou-se diferenças estatisticamente significativas entre os dias $1 \mathrm{e}$

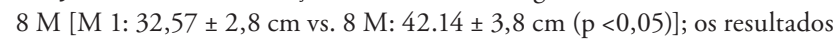
mostraram que os valores diminuíram em SAR (M 1: 13,14 $\pm 12,27$ cm-8 $\mathrm{m}: 6,42 \pm 7,36 \mathrm{~cm}$ ); Finalmente, observamos diferenças significativas entre $1 \mathrm{M}$ e $8 \mathrm{M}$ em CNT [M 1: $10,36 \pm 1,7$ vs paliers $8 \mathrm{M}: 12,5 \pm 1,4$ driveshafts; $(\mathrm{P}<0,05)]$. Conclusóes. Melhorias significativas foram observadas capacidades neuromusculares, potência aeróbica e flexibilidade.

Palavras-chave: basquetebol, física, jovem.

\section{Introducción}

El baloncesto (B), uno de los deportes más populares (Ostojic et al 2006), se caracteriza por una alta heterogeneidad entre equipos y practicantes (Bolonchuk et al, 1991). En los

Dirección para correspondencia [Correspondence address]: Julio CallejaGonzález. Departamento de Educación Física y Deportiva. Facultad de Ciencias de la Actividad Física y el Deporte. Universidad del País Vasco (UPV/EHU). Lasarte Ataria, 71. CP 01007. Vitoria (Espańa). E-mail: julio.calleja@ehu.es últimos ańos se han publicado numerosos estudios descriptivos (Hoffman et al, 1999a) sobre fisiología (Smith, 1991), recuperación (Delextrat, 2013), perfil hormonal (Schelling et al, 2009), antropometría (Drinkwater et al, 2008), características neuromusculares (Ben Abdelkrim et al, 2007; Delextrat et al, 2012; Stojanovic et al, 2012), percepción de esfuerzo (Fuentes et al, 2013) en base a diferentes posiciones (PonceGonzález et al, 2015) o relación entre condición física y aspec- 
tos tácticos (Leite et al, 2014) en B. Algunos de ellos se realizaron con jugadores de élite de países con muchas tradición en B, como Serbios (Ostojic et al, 2006), Franceses (Sallet et al, 2005) e Israelíes (Hoffman et al, 1999b), y otros, como Indúes (Sodhi, 1980), donde el B no es un deporte relevante, Sin embargo, pocos estudios han analizado la evolución de la condición fisica en $\mathrm{B}$, ya sea con jugadores de élite (Ostojic et al, 2006), o con jóvenes en etapas de transición (Hoare et al, 2000). Por este motivo, el objetivo de nuestro trabajo fue analizar la evolución de las capacidades físicas de jugadores de baloncesto de medio nivel durante 2 años, más concretamente en la transición de la categoría cadete a junior.

\section{Método}

\section{Participantes}

15 jugadores de baloncesto no profesionales pertenecientes a un club de división autonómica (2001-03 tomaron parte en el estudio (Edad Inicial $=14 \pm 0$ años; Masa $=73.8 \pm 9.1 \mathrm{~kg}$; Altura $=195.5 \pm 3 \mathrm{~cm}$; \% graso $=9 \pm 2.3 \%$; Experiencia en B: $2 \pm 1$ años). El grupo incluía 2 bases, 3 escoltas, 5 aleros, 2 ala pívots y 3 pivots. Durante el estudio, su programa de entrenamiento se componía de 4 sesiones semanales técnicas + 2 de preparación fisica de 120 min y un partido. Todos ellos dieron su consentimiento informado a participar voluntariamente en el estudio. Los procedimientos utilizados en los participantes y controles han sido realizados tras obtención de un consentimiento informado de los mismos, acorde a la Declaración de Helsinki (2008).

\section{Protocolo}

Los jugadores fueron testados durante los dos ańos consecutivos correspondientes al último ańo de la etapa cadete y al primer año de la etapa junior. Todos los test fueron desarrollados en 4 momentos (M) en cada una de las 2 temporadas [(1. Septiembre (pre-temporada), 2. Diciembre, 3. Abril y 4. Julio (post-temporada)], para un total de $8 \mathrm{M}$. Se realizaron las siguientes pruebas: test de $20 \mathrm{~m}$ sprint (MST), test de agilidad T (AGIT), lanzamiento de balón medicinal de $5 \mathrm{~kg}$ (5KGT), saltos en contra movimiento (CMJ), test de flexibilidad Sit and Reach (SAR) y la C. Navette (CN). Los sujetos realizaron $10 \mathrm{~min}$ de calentamiento, series de movimientos dinámicos (e.g. cambios de dirección, skipping) y ejercicios de prevención lesional.

Batería de Test

MST TEST. Se realizó un MST 3 veces con 2 min de recuperación. Se colocaron fotocélulas (Newtest OY, Oulu, Finland) a una altura de $0.5 \mathrm{~m}$ sobre el suelo. El jugador se colocaba $0.5 \mathrm{~m}$ antes de la línea de salida. El tiempo fue activado cuando el sujeto pasó la primera célula. El coeficiente de prueba test-retest fue de 1.8 (Hoffmann et al, 1996). AGIT TEST. El test AGIT ha sido utilizado en B ya que reproduce los movimientos del juego (Jukic et al, 1999). El sujeto realiza un sprint de salida y en línea recta a un cono colocado a $9 \mathrm{~m}$ y después a otro colocado a $4.5 \mathrm{~m}$ a su lado izquierdo sin cruzar los pies. Después de éste, hacia el lado derecho hasta un tercer cono a $9 \mathrm{~m}$, y vuelta al cono del medio y hacia atrás a la posición inicial Se anotó el mejor de los dos tiempos. 5KGT. El test valora la potencia muscular en tren superior (Izquierdo et al, 2002). El sujeto detrás de la línea marcada debe impulsar el balón de $5 \mathrm{~kg}$ por encima de la cabeza tan lejos como sea posible. Se anota el mejor de 3 intentos. CMJ TEST. El test de CMJ fue realizado mediante una plataforma de contactos (Ergojump, Globus Inc. Italy). Los sujetos comenzaron la prueba en posición anatomica con las manos en la cintura y ambos pies juntos (Bosco, 1983). Debían saltar los más alto posible. Se realizaron 3 saltos separados por $2 \mathrm{~min}$. Se registró el mejor salto. El CMJ se caracteriza por una baja variabilidad entre tests (CV 3.0\%) (Markovic et al, 2004). SAR TEST. El SAR es un test utilizado para evaluar la flexibilidad de tronco (Hui and Yuen, 2000), presentando una validez y fiabilidad concurrente (Baltaci et al, 2003) considerado un test moderadamente válido con correlaciones de 0.39 to 0.76 . CN TEST. La prueba fue evaluada mediante el protocolo de Leger (Leger y lambert, 1982). Los sujetos, relizaron el test de lado a lado de la cancha con 20 metros de separación entre ambas lineas, mientras el audio marcaba los tiempos de paso. La velocidad inicial fue de $8.5 \mathrm{~km} / \mathrm{h}$, incrementandose 0.5 $\mathrm{km} / \mathrm{h}$ por min. Se midió en palieres $(\mathrm{p})$.

\section{Analisis Estadistico:}

Los datos se presentan como media \pm DS. Se realizó una prueba de normalidad, Shapiro -Wilk (<15), y homogeneidad de la varianzas mediante Levene. Se optó por pruebas no paramétricas. Se utilizó el test de Mann-Whitney U para determinar diferencias significativas entre el primer y último momento. Para las medidas repetidas se aplicó una prueba de Friedman. El nivel de significación fue aceptado para una $p<0.05$. Se utilizó el paquete estadístico SPSS 20.0, Chicago, EE.UU).

\section{Resultados}

Un total de $8 \mathrm{M}$ fueron registrados durante dos años (cadete y junior) (Tabla 1).

MST. Se observó un incremento en el MST entre el $1^{\circ} \mathrm{M}$ y el $8^{\circ} \mathrm{M}\left(1^{\circ} \mathrm{M}: 10.36 \pm 1.79 \mathrm{~m} / \mathrm{s}\right.$ vs. $8^{\circ} \mathrm{M}: 12.50 \pm$ $1.44 \mathrm{~m} / \mathrm{s}$ ).

En el $2^{\circ} \mathrm{M}$ la media fue $11.64 \pm 1.72 \mathrm{~m} / \mathrm{s}$, en el $3^{\circ} \mathrm{M}$ : $12.00 \pm 1.65 \mathrm{~m} / \mathrm{s}$ 
En el $4^{\circ} \mathrm{M}: 12.43 \pm 1.42 \mathrm{~m} / \mathrm{s}$, en el $5^{\circ} \mathrm{M}: 10.43 \pm 2.21$ $\mathrm{m} / \mathrm{s}$, en el $6^{\circ} \mathrm{M}: 11.71 \pm 1.91 \mathrm{~m} / \mathrm{s}$, en el $70 \mathrm{M}: 12.00 \pm$ $1.80 \mathrm{~m} / \mathrm{s}$.

Los valores medios, durante el periodo cadete y junior en el MST fueron $11.60 \mathrm{~m} / \mathrm{s}$ y $11.66 \mathrm{~m} / \mathrm{s}$ respectivamente sin diferencias significativas.

Observamos mejoras significativas en el $4^{\circ} \mathrm{M}$ respecto al $1^{\circ}\left[1^{\circ} \mathrm{M}: 3.16 \pm 0.27 \mathrm{~m} / \mathrm{s}\right.$ Vs. $4^{\circ} \mathrm{M}: 2.87 \pm 0.17 \mathrm{~m} / \mathrm{s}$; $(\mathrm{p}<0.05)]$ (Tabla 1$)$.

AGIT. No se observaron diferencias significativas.

5KGT. No se describió diferencia en ningún momento.

CMJ. El test presentó mejoras en $\left(1^{\circ} \mathrm{M}: 32.57 \pm 2.82 \mathrm{~cm}\right.$ respecto al $\left.8^{\circ} \mathrm{M}: 43.14 \pm 3.80 \mathrm{~cm}\right)$. En el $2^{\circ} \mathrm{M}$ se obtuvieron medias de $35.14 \pm 2.73 \mathrm{~cm}$, en el $3^{\circ} \mathrm{M}: 36.86$ $\pm 2.47 \mathrm{~cm}$

En el $4^{\circ} \mathrm{M}: 38.00 \pm 3.6 \mathrm{~cm}$, en el $5^{\circ} \mathrm{M}: 37.29 \pm 4.53 \mathrm{~cm}$ En el $6^{\circ} \mathrm{M}: 39.86 \pm 3.71 \mathrm{~cm}$

El 7o M: $41.86 \pm 3.80 \mathrm{~cm}$. Los valores medios del periodo cadete fueron de $35.70 \mathrm{~cm}$ y los del junior: $41.59 \mathrm{~cm}$. Se observaron diferencias significativas entre el $1^{\circ} \mathrm{M}$ y el $8^{\circ} \mathrm{M}\left[1^{\circ} \mathrm{M}: 37 \pm 01 \mathrm{~cm} \mathrm{Vs.} 8^{\circ} \mathrm{M}: 43 . \pm 15 ;(\mathrm{p}<0.05)\right]$ (Tabla 1).
SAR. Los valores decrecieron ( $1^{\circ} \mathrm{M}: 13.14 \pm 12.27 \mathrm{~cm} \mathrm{Vs}$. a $\left.8^{\circ} \mathrm{M}: 6.42 \pm 7.36 \mathrm{~cm}\right)$.

En el $2^{\circ} \mathrm{M}$ los valores fueron $13.86 \pm 11.71 \mathrm{~cm}$

En el $3^{\circ} \mathrm{M}: 6.70 \pm 7.11 \mathrm{~cm}$.

En el $4^{\circ} \mathrm{M}: 6.57 \pm 7.48 \mathrm{~cm}$

En el $5^{\circ} \mathrm{M}: 6.71 \pm 7.01 \mathrm{~cm}$

En el $6^{\circ} \mathrm{M}: 7.28 \pm 7.11 \mathrm{~cm}$

En el $7{ }^{\circ} \mathrm{M}: 6.57 \pm 7.80 \mathrm{~cm}$.

Los valores medios durante el periodo cadete fueron de $10.06 \mathrm{~cm}$ y en junior $6.87 \mathrm{~cm}$.

CNT. EL CNT mejoró, presentando diferencias significativas entre momentos [(10 M: $10.36 \pm 1.7 \mathrm{n} \mathrm{Vs.} 8^{\circ} \mathrm{M}$ : $12.50 \pm 1.44 \mathrm{n}) ;(\mathrm{p}<0.05)]$.

En el $2^{\circ} \mathrm{M}$ los valores medios fueron de $11.64 \pm 1.72 \mathrm{p}$. En el $3^{\circ} \mathrm{M}: 12.00 \pm 1.65 \mathrm{p}$.

En el $4^{\circ} \mathrm{M}: 12.43 \pm 1.42 \mathrm{p}$.,

En el $5^{\circ} \mathrm{M}: 10.43 \pm 2.22 \mathrm{p}$.,

En el $6^{\circ} \mathrm{M}: 11.71 \pm 1.91 \mathrm{p}$.,

En el $7^{\circ} \mathrm{M}: 12.00 \pm 1.80 \mathrm{p}$.

Los valores medios en el periodo de categoría cadete fueron de $11.60 \mathrm{p}$ y en junior de $11.66 \mathrm{p}$.

Tabla 1. Evolución de las capacidades en 8 momentos durante 2 años y diferencias entre (periodo Cadete -Junior ).

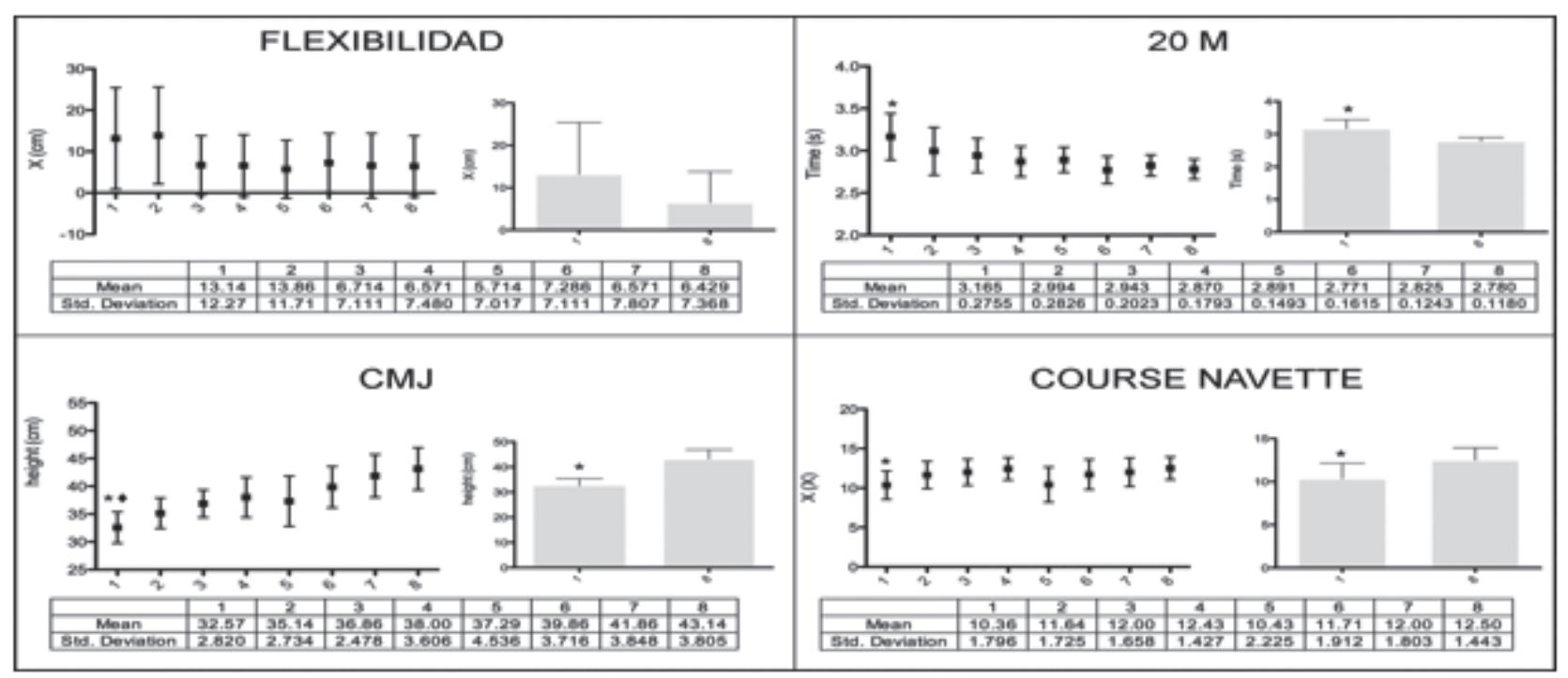

\section{Discusión}

Este estudio fue diseñado para analizar la evolución de ciertos parametros condicionales en jugadores de medio nivel cadetes y junior ya que, para nuestro conocimiento, no hay investigaciones que describan la evolución de las capacidades condicionales en esta población. Nuestros resultados conclu- yen que tras 2 años de entrenamiento sistemático, las capacidades neuromusculares, la potencia aeróbica y la flexibilidad mejoraron de manera significativa mientras que la agilidad y la fuerza no presentaron cambios significativos. La especificidad del MST en B debiera tenerse en cuenta no sólo por los desplazamientos parecidos producidos durante el juego (Delextrat and Cohen, 2008), sino también por la alta co- 
rrelación demostrada con el rendimiento en B (Hoffman et al, 1996). El mejor resultado observado fue en diciembre del $1^{\circ}$ año (cadete) $2.87 \pm 0.17 \mathrm{~m} / \mathrm{s}$, quedando justificado con análisis discriminantes previos entre jugadores de élite y noélite, revelando que la velocidad es un parámetro importante en la identificación de talentos (Mohamed et al, 2009). El $\mathrm{CMJ}$ mejoró significativamente del $1^{\circ} \mathrm{M}(32.57 \pm 2.8 \mathrm{~cm}) \mathrm{al}$ $8^{\circ} \mathrm{M}(43.14 \pm 15 \mathrm{~cm} ; \mathrm{p}<0.05)$. A pesar de las limitaciones de este test ( $\mathrm{Ziv}$ et al, 2009), está demostrado que 10 semanas de entrenamiento con cargas de volumen e intensidad moderada incrementan el salto en jugadores adolescentes (Santos y Janeira, 2011). El SAR es una prueba para evaluar la flexibilidad de la parte posterior de la pierna y la musculatura isquiotibial descrita por Christou et al, (2006) y que es utilizada como valoración de la flexibilidad. No existe evidencia en $\mathrm{B}$ acerca de la evolución de esta capacidad, sin embargo las directrices del ACSM concluyen que es un test con valores poco consistentes en menores de 16 años (ACSM, 2006). Nuestros resultados demostraron que los valores decrecieron (1०M: $13.14 \pm 12.27 \mathrm{~cm}$ a $\left.8^{\circ} \mathrm{M}: 6.42 \pm 7.36 \mathrm{~cm}\right)$, presentando datos que no podemos comparar dada la inexistencia de otros trabajos en $\mathrm{B}$. El sistema aeróbico es una fuente energética durante los partidos, sin emabrgo, otros autores enfatizan la importancia de la potencia anaeróbica (Delextrat and Cohen, 2008). En cualquier caso, presentar unos niveles adecuados de capacidad aeróbica es un objetivo para la mejora en la condición fisica en jovenes deportistas. Nuestros datos presentaron diferencias significativas a largo plazo $(10.36 \pm 1.79$ p Vs. $12.50 \pm 1.44$ p). El mejor resultado en este test tuvo lugar en junio, en el $8^{\circ} \mathrm{M}$, al fnal del año de junior con un tiempo de12.50 \pm 1.4 p con una edad media de los jugadores de 16 años. El incremento del $\mathrm{VO}_{2 \max }$ en la edad pubertal se corresponde con el mayor incremento de altura que junto con otros muchos cambios organicos incrementa la capacidad de resistencia (Kobayasshi, 1978).

\section{Conclusión}

Las capacidades neuromusculares, las respuestas cardiovasculares y la flexibilidad mejoraron en jugadores de baloncesto cadetes y junior de medio nivel tras dos años de entrenamiento sistematico. Sin embargo, en las pruebas de agilidad y fuerza del tren superior, no obervamos cambios significativos.

\section{Aplicaciones practicas}

En base a os resultados obtenidos los preparadores físicos y entrenadores de baloncesto debieran aplicar contenidos de entrenamiento aplicados al desarrollo del potencial neuromuscular, así como a la capacidad de resistencia general y la flexibilidad con deportistas entre los 14 y los 16 años.

\section{Referencias}

1. American College of Sports Medicine (2006). ACSM's Guidelines for Exercise Testing and Prescription ( $7^{\text {th }}$ Edition). Lippincott Williams and Wilkins: Philadelphia.

2. Baltaci, G., Un, N., Tunay, V., Besler, A., and Gerçeker, S. (2003). Comparison of three different sit and reach tests for measurement of hamstring flexibility in female university students. British Journal Of Sports Medicine, Feb, 37 (1), 59-61.

3. Ben Abdelkrim, N., El Fazaa, S., and El Ati, J. (2007). Time-motion analysis and physiological data of elite under-19-year-old basketball players during competition. British Journal Of Sports Medicine, Feb, 41 (2), 69-75.

4. Bolonchuck, W., Lukaski, H., and Siders, W. (1991). The structural, functional, and nutritional adaptation of college basketball players over a season. Journal Of Sports Medicine and Physical Fitness, Jun 31 (2), 165-172.

5. Bosco, C., Luhtanen, P., and Komi, PV. (1983) A simple method for measurement of mechanical power in jumping. European Journal Applied and Occupational Physiology, 50 (2), 273-282.

6. Christou, M., Smilios, I., Sotiropoulos. K., Volaklis. K., Pilianidis, T., Tokmakidis. S.P. (2006). Effects of resistance training on the physical capacities of adolescent soccer players. J Strength Cond Res. Nov;20(4):783-791.

7. Delextrat, A., Trochym, E., Calleja-González, J. (2012) Effect of a typical in-season week on strength jump and sprint performances in national-level female basketball players. Journal of Sports Medicine and Physical Fitness, Apr; 52 (2),128-136.

8. Delextrat, A., Calleja-González J., Hippocrate, A., Clarke, ND. (2013) Effects of sports massage and intermittent cold-water immersion on recovery from matches by basketball players. Journal of Sports Sciences, 31(1),11-19.

9. Drinkwater, EJ., Pyne, DB., and McKenna, MJ. (2008). Design and interpretation of anthropometric and fitness testing of basketball players. Sports Medicine, 38 (7), 565-578.

10. Fuentes Azpiroz, M., Feu, S., Jiménez, C., and Calleja-González, J. (2013). Perceived exertion effort in mini basketball players and its relationship with training volume. Revista de Psicología del Deporte. Vol 22, No (1), 205-208.

11. Hoare, D.G. (2000). Predicting success in junior elite basketball players- the contribution of anthropometric and physiological attributes. Journal Of Science and Medicine in Sport. Dec, 3, (4), 391-405.

12. Hoffman, J., Epstein, S., Einbinder, S., and Weinstein, Y. (1999a). The influence of aerobic capacity on anaerobic performance and recovery indices in basketball players. Journal Of Strength and Conditioning Research, 13, (3), 280-285.

13. Hoffman, J., Epstein, S., Yarom, CY., Zigel, L., and Einbinder, M (1999b). Hormonal and biochemical changes in elite basketball players during a 4-week training camp. Journal Of Strength and Conditioning Research, 13, (3), 280-285.

14. Hoffman, J.R., Tenenbaum, G., Maresh, CM., and Kraemer, WJ (1996). Relation between athletic performance tests and playing time in elite collegue basketball players. Journal Of Strength and Conditioning Research, 10, (2), 67-71.

15. Hui, SS., and Yuen, PY. (2000). Validity of the modified back-saver sitand-reach test: a comparisn with other protocols. Medicine and Science in Sports and Exercise, Sep, 32, (9), 1655-1659.

16. Izquierdo, M., Häkkinen, K., Gonzalez-Badillo, JJ., Ibáñez, J., and 
Gorostiaga, EM. (2002). Effects of long-term training specificity on maximal strength and power of the upper and lower extremities in athletes from different sports. European Journal Of Applied Physiology, Jul, 87, (3), 264-271.

17. Jukić, I., Milanović, D., and Vuleta, D. (1999). Analysis of changes in indicators of functional and motor readiness of female basketball players within one-year training cycles. Coll Antropolog, Dec, 23, (2), 691-706.

18. Kobayashi, K. (1978). Aerobic power as related to by growth and training in japanese boys, a longitudinal study. Journal Of Applied Physio$\log y, 44,(5), 666-672$.

19. Léger, LA., and Lambert, J. (1982). A maximal multistage $20 \mathrm{~m}$ shuttle run test to predict $\mathrm{VO}_{2 \max }$. European Journal Of Applied Physiology, Vol $49,1-5$.

20. Leite, N.M., Leser, R., Gonçalves. B., Calleja-Gonzalez. J., Baca. A., Sampaio. J. (2014).

21. Effect of defensive pressure on movement behaviour during an under-18 basketball game. International Journal Of Sports Medicine, Aug;35(9),743-748.

22. Markovic, G., Dizdar, D., Jukic, I., and Cardinale, M. (2004). Reliability and factorial validity of squat and countermovement jump tests. Journal Of Strength and Conditioning Research, Aug, 18, (3), 551-555.

23. Mohamed, H., Vaeyens, R., Matthys, S., Multael, M., Lefevre, J., Lenoir, M., and Philppaerts, R. (2009). Anthropometric and performance measures for the development of a talent detection and identification model in youth handball. Journal Of Sports Science, Feb, 1, 27,(3), 257-266.

24. Ostojić, SM., Mazic, S., and Dikic, N. (2006). Profiling in basketball: physical and physiological characteristics of elite players. Journal of Strength and Conditioning Research, Nov, 20, (4), 740-7444.

25. Pacheco, L., Balius, R., Aliste, L., Pujol, M., and Pedret, C. (2011).
The acute effects of different stretching exercises on jump performance. Journal Of Strength and Conditioning Research, Nov, 25, (11), 29912998.

26. Ponce-González, J.G., Olmedillas, H., Calleja-González, J., Guerra, B., Sanchis-Moysi, J. (2015). Physical fitness, adiposity and testosterone concentrations are associated to playing position in professional basketballers. Nutrición Hospitalaria. Jun 1;31(n06), 2624-2632.

27. Sallet, P., Terrier, D., Ferret., M., Vitelli, V., and Baverel, G. (2005). Physiological differences in professional basketball players as a function of playing position and level of play. Journal Of Sports Medicine and Physical Fitness, Sep, 45,(3), 291-294.

28. Santos, EJ., and Janeira, MA. (2012).The effects of resistance training on explosive strength indicators in adolescent basketball players. Journal Of Strength and Conditioning Research, Oct, 26,(10), 2641-2647.

29. Schelling i del Alcázar, X., Calleja-González, J., and Terrados Cepeda, N. (2009). Análisis hormonal en un equipo de baloncesto de élite durante una temporada. Revista de Psicología del Deporte. 18, (3), 363-367.

30. Smith, H., and Thomas, S.G. (1991). Physiological characterise of elite female basketball players. Canadian Journal Of Sport. Sciences/ Revue Canadiense Des Sciences Du Sport (Champaign,-I11), Dec,16, (4), 289295.

31. Sodhi, H.S. (1980). Kinanthropometry and performance of top ranking Indian basketball players. British Journal Of Sports Medicine, Jul,14, (2-3), 139-144.

32. Stojanovic, M.D., Ostojic, S.M., Calleja-González, J., Milosevic, Z., Mikic, M. (2012) Correlation between explosive strength, aerobic power and repeated sprint ability in elite basketball players. Journal of Sports Medicine and Physical Fitness, Aug; 52(4), 375-381.

33. Ziv, G., and Lidor, R. (2009). Physical attributes, physiological characteristics, on-court performances and nutritional strategies of female and male basketball players. Sports Medicine, 39, (7), 547-68. 